\title{
Independência do Banco Central e coordenação de políticas: vantagens e desvantagens de duas estruturas para estabilização
}

\author{
Central Bank independence and policy coordination: advantages \\ and disadvantages of two structures for stabilization
}

HELDER FERREIRA DE MENDONÇA*

RESUMO: Hoje em dia, a sabedoria convencional ortodoxa acredita que a adoção de um banco central independente melhora o alinhamento da política fiscal com a política monetária e, assim, aumenta a coordenação entre as autoridades fiscais e monetárias. No entanto, a ideia de coordenação não pode ser reduzida a isso. Este trabalho faz uma breve análise sobre as vantagens e desvantagens da independência da proposta e coordenação do banco central entre as políticas monetária e fiscal. Os resultados denotam que a coordenação de políticas é uma estrutura melhor para alcançar os diversos objetivos macroeconômicos.

PALAVRAS-CHAVE: Independência do banco central; coordenação; políticas macroeconômicas.

ABSTRACT: Nowadays, the orthodox conventional wisdom believes that the adoption of an independent central bank improves the alignment of fiscal policy with monetary policy, and thus, increases the coordination between fiscal and monetary authorities. However, the idea of coordination cannot be reduced to this. This paper makes a brief analysis concerning the advantages and disadvantages that belong to the independence of the central bank proposition and coordination between monetary and fiscal policies. The findings denote that the coordination of policies is a better framework to achieve the several macroeconomic goals. KEYWORDS: Independence of central bank; coordination; macroeconomic policies. JEL Classification: E52; E58; E61.

\section{INTRODUÇÃO}

No período anterior aos anos 70 prevalecia a idéia, tipicamente keynesiana, de que a política monetária deveria ser conduzida tendo a preocupação de evitar o

\footnotetext{
* Professor do Departamento de Economia da Universidade Federal Fluminense - UFF, Niterói/RJ, Brasil. E-mail: helderfm@hotmail.com. Submetido: 20/Março/2002; Aprovado: 14/maio/2002.
} 
desemprego. É importante ressaltar que este comportamento não significava que a inflação era desprezada como um dos objetivos da política monetária, o ponto a ser lembrado é que, naquela época, a inflação não representava uma ameaça para o equilíbrio macroeconômico dos diversos países e havia a necessidade de recuperar, via crescimento econômico, o tempo perdido com a II Grande Guerra Mundial. Em termos teóricos, pode-se dizer que a estrutura desenvolvida por Tinbergen (1952) e Mundell (1960) - no qual era admitido o efeito da política monetária sobre o produto e a inflação - era válida.

Todavia, diversos fatores (no período 1971-1973) contribuíram de forma decisiva para o rompimento da estrutura que prevalecia na condução da política monetária. Do ponto de vista prático foram observados o fim do sistema de Bretton Woods e o primeiro grande choque do petróleo. Por outro lado, sob a perspectiva teórica, foi introduzido o conceito de expectativas racionais à teoria da política econômica. A conjugação desses fenômenos foi responsável por uma inflexão do desenho da política monetária em busca da estabilidade de preços.

Diante do cenário que prevalecia no início dos anos 70, a interpretação friedmaniana de que a política monetária teria seus efeitos reais confinados ao curto prazo despertou o interesse dos economistas para o uso de regras (expansão monetária $a d$ hoc) como um mecanismo útil para garantir a estabilidade econômica. Apesar disso, sob a perspectiva teórica, era preciso encontrar um novo argumento que justificasse a incoerência do uso da política monetária com o objetivo de causar efeitos reais sobre a economia. A variável-chave encontrada foi a credibilidade da política monetária. A partir de então, diversos estudos foram elaborados com a necessidade de satisfazer duas condições no equilíbrio (Barro, 1986):

i) a validade do preceito das expectativas racionais, isto é, as expectativas in-

flacionárias dos agentes são corretas na média; e ii) embora o responsável pela política tenha o poder, em cada período, de iludir o público via surpresa inflacionária, ele não é motivado a fazer uso deste mecanismo. Em outras palavras, no equilíbrio - o benefício marginal de uma inflação surpresa deve ser igual ao custo marginal da inflação.

Este artigo tem por objetivo fazer uma análise sucinta das principais vantagens e desvantagens provenientes da independência e coordenação de políticas macroeconômicas. Além desta introdução, o trabalho apresenta-se estruturado da seguinte forma: a segunda seção apresenta os motivos que levaram à proposição de independência do Banco Central (IBC) e as principais vantagens e desvantagens relacionadas a um banco central independente (BCI); a terceira seção apresenta a justificativa teórica para a coordenação de políticas macroeconômicas e os prós e contras da adoção desta estrutura; por último é apresentada a conclusão dos pontos levantados ao longo do artigo. 


\section{VANTAGENS E DESVANTAGENS DA IBC}

A essência teórica que sustenta a proposição de IBC consiste na neutralidade da moeda. ${ }^{1}$ Assim, sob esta perspectiva, o BC deveria abandonar a tentativa de uso da política monetária na busca de efeitos reais, pois seria apenas uma fonte de ruído que prejudicaria a interpretação correta dos agentes. Em outras palavras, a antiga estrutura que admitia a possibilidade da política monetária afetar o produto e a inflação reduziu-se à busca da estabilidade de preços.

O esquema 1 sumaria os principais argumentos responsáveis pela origem da proposição de IBC. Antes do início da década de 70 prevalecia a idéia de que a política monetária (instrumento) poderia estar relacionada a mais de um objetivo (metas), isto é, a estrutura apresentada por Tinbergen (1952) seria adequada para a formulação da política econômica. Em geral, era bem aceito pelos economistas que a política monetária poderia ser usada de forma contracíclica sendo capaz de afetar variáveis reais (produto) e nominais (inflação). Entretanto, o fim do Sistema de Bretton Woods e o primeiro grande choque do petróleo fizeram com que a busca por mecanismos que fossem capazes de combater a inflação ganhasse destaque. Concomitante à necessidade de natureza prática por um novo desenho da política monetária, a introdução do conceito de expectativas racionais à análise da política econômica por Lucas $(1972,1973)$ e Sargent $(1973)$ fez com que diversos economistas passassem a assumir a impossibilidade da política monetária causar efeitos reais sobre a economia, o que por conseqüência, levou ao fortalecimento do pressuposto da neutralidade da moeda.

Esquema 1

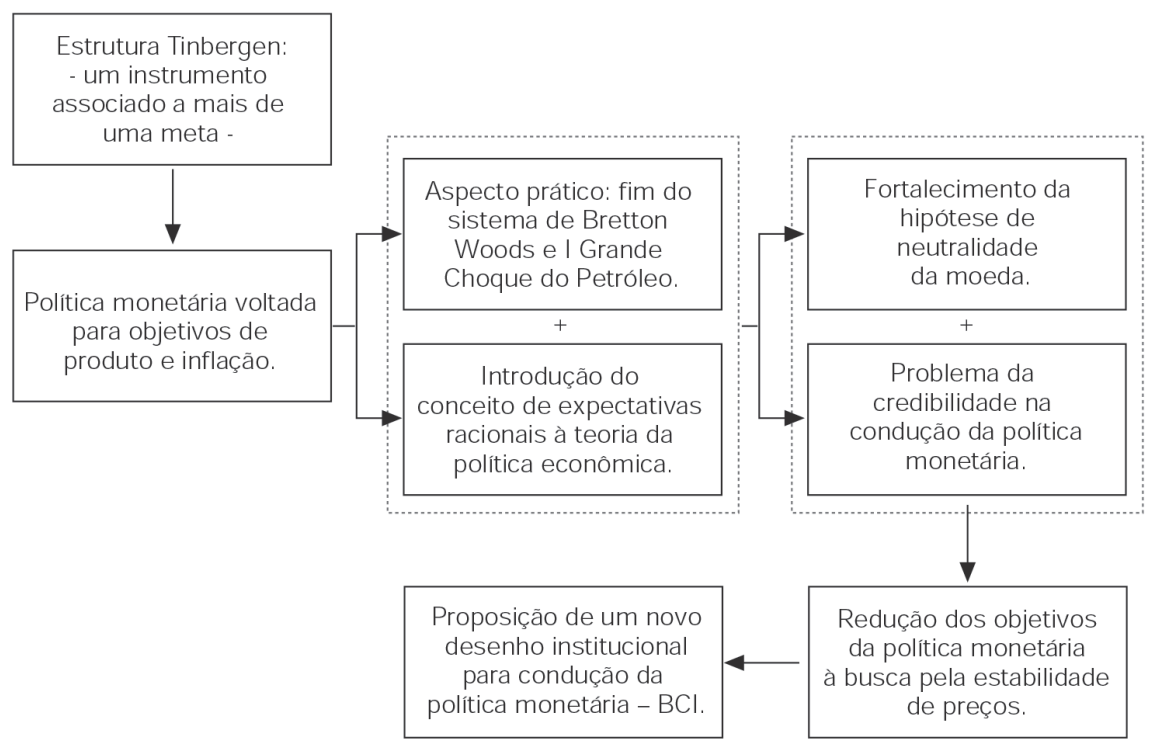

${ }^{1}$ Para uma análise sobre a teoria da IBC, ver Mendonça 2000. 
Além das observações acima, o problema da credibilidade das autoridades na condução das políticas — motivado pelos estudos de Kydland \& Prescott (1977) e Barro \& Gordon (1983) — culminou com a necessidade de serem desenvolvidas estruturas para a política monetária que tivessem como preocupação básica a estabilidade de preços. A partir deste conjunto de restrições, Rogoff (1985) elaborou o artigo que serve como referência básica para a literatura sobre IBC.

A literatura sobre BCIs tem início a partir da identificação do viés inflacionário que está presente na condução da política monetária. A teoria moderna sobre credibilidade atribui a existência do viés inflacionário à inconsistência dinâmica da política monetária no modelo expectacional da curva de Phillips de determinação do produto ou do imposto inflacionário. Nesse sentido, um BCI seria capaz de comprometer-se com uma inflação baixa sem sofrer pressões do governo para o financiamento de déficits. Ou seja, um BCI seria capaz - de acordo com seus defensores - de estabilizar o nível de preços da economia e ainda disciplinar os gastos do governo.

De acordo com a literatura que discute a IBC diversos efeitos sobre a economia podem ser percebidos após sua implementação. ${ }^{2} \mathrm{O}$ mais propalado resultado de um BCI refere-se ao fato de que a implementação de uma autoridade monetária independente seria capaz de garantir uma taxa de inflação menor e mais estável para o país que adotasse essa estrutura. Apesar deste resultado ser a evidência empírica mais referida pelos defensores de um BCI, deve-se ressaltar que tais evidências não indicam que este resultado seja válido para o caso dos países emergentes.

Cukierman (1994) destaca o fato de que a experiência de países que convivem com elevada inflação tem demonstrado que a conquista da estabilidade tem sido obtida via desenvolvimento. Portanto, tal objetivo não pode ser alcançado somente pela delegação de autoridade para o BC, uma vez que a discussão sobre BCIs não depende apenas do aspecto econômico, mas também do político. O que pode ser ponderado é que delegar autoridade para o BC, de forma a tornar seu compromisso com a inflação crível pelo público, constitui-se em um instrumento que pode funcionar como efeito preventivo na repetição de episódios que favoreçam ao incremento da inflação.

Outra importante observação para BCIs refere-se à possível mudança de comportamento da autoridade monetária quanto à determinação da taxa de juros. As evidências empíricas, presentes na literatura, mostram que não há uma clara relação que possa ser encontrada entre a independência e a média das taxas de juros reais ex-post. Esta é uma constatação importante porque traz implicações significativas para avaliar os impactos de um BCI sobre o crescimento econômico. Para esta análise, duas visões opostas têm sido seguidas:

\footnotetext{
${ }^{2}$ A discussão aqui realizada é feita com base na literatura padrão sobre IBC, o objetivo é mostrar quais as benesses decorrentes da implementação de um BCI sob a interpretação de seus defensores. Para o leitor interessado em observar as evidências empíricas relacionadas à IBC, ver Alesina \& Summers (1993) e Mendonça (1998).
} 
i) taxa de juros reais depende do crescimento monetário - um baixo nível de inflação causado pelos resultados de uma política monetária restritiva e pela elevação das taxas de juros reais pode ter efeitos negativos sobre o nível de investimentos, e portanto, no crescimento econômico; e

ii) BCIs como fomentador do crescimento econômico - dado que um BCI é menos propenso a pressões políticas, suas ações podem ser realizadas de forma mais prognosticada, o que contribui para maior estabilidade econômica e crescimento.

Deve-se fazer algumas considerações em relação ao segundo ponto sobredito. Boa parte dos economistas tem sustentado a hipótese de que a inflação é prejudicial para o crescimento econômico, uma vez que o efeito direto é um aumento do nível de incerteza. Como é admitido que um BCI é capaz de garantir uma menor variabilidade da inflação, é esperado, ao menos no plano teórico, que com a redução da incerteza sobre a inflação os agentes possam aumentar a capacidade de planejamento. A conseqüência desta observação é que haveria um aumento do investimento da economia acarretando um aumento da demanda agregada. Em outras palavras, sob esta interpretação, um BCI poderia ter um efeito positivo para o crescimento econômico.

A despeito do argumento acima se mostrar razoável, as evidências empíricas não dão suporte à idéia de que poderia haver um aumento do crescimento econômico em virtude de uma maior IBC (Hahn e Sturm, 1992). Um elemento que pode justificar o porquê de não haver uma relação clara entre o crescimento e a IBC, advém do fato de que, por um lado, uma maior estabilidade da economia poderia realmente incentivar o investimento privado, mas por outro, a IBC poderia reduzir o papel empreendedor do estado por não permitir o financiamento de seus gastos. As evidências empíricas denotam que BCs considerados com elevado grau de independência, como é o caso do alemão, suíço e do americano não financiam déficits. ${ }^{3}$

Trazendo-se para a discussão a proposta de preanúncios para o comportamento das políticas econômicas, aparece a questão relativa à transparência ou controle sobre as metas pretendidas. Sob este aspecto, duas possibilidades extremas podem ser usadas para ilustrar os trade-offs envolvidos. Em um extremo, o BC poderia comprometer-se com a meta referente à base monetária e, de outro, com as metas inflacionárias. No primeiro caso, a precisão do controle é alta desde que a base seja parte das obrigações do banco sobre a qual o controle é perfeito. Entretanto, devido à conexão tênue entre a base e o nível de preços, esta relação não é muito transparente, sobretudo para agentes que não realizam operações constantes nos mercados financeiros. Por outro lado, uma meta que envolva uma taxa de inflação ou nível de preços, é muito transparente para os agentes desde que eles possam observar o resultado final (por exemplo, se houve ou não aumento dos preços). Entretanto, o con-

\footnotetext{
${ }^{3}$ Hahn e Sturm (1992) verificaram que o crédito concedido ao Governo é influenciado pelo grau de independência dos bancos centrais e, ainda, que o tamanho do déficit público é afetado pelo mesmo motivo.
} 
trole do BC nesse caso é imperfeito, pois de forma diferente do domínio que ele pode exercer sobre a base monetária, seu controle sobre a inflação não é direto.

Um outro ponto que não deve ser desprezado quando se pondera as implicações decorrentes da adoção de um BCI refere-se à questão democrática. Em geral, a escolha dos responsáveis pela condução da política monetária não é feita de forma direta pelo público, o que por conseguinte, revela uma estrutura não-democrática. A justificativa para este procedimento, segundo os defensores de um BCI, advém do fato de o público ser míope em relação ao bem-estar social. Sob esta interpretação, haveria uma tendência de os agentes pressionarem o governo para a obtenção de um nível de emprego mais elevado, gerando uma pressão capaz de estimular o viés inflacionário na condução da política monetária no caso de o BC ser subserviente ao governo. ${ }^{4}$

A partir da literatura padrão elabora-se, a seguir, um quadro (tabela 1) que sumaria as principais vantagens e desvantagens relacionadas à adoção de um BCI.

Tabela 1: Principais vantagens e desvantagens da adoção de um $\mathrm{BCl}$

\begin{tabular}{ll}
\hline \multicolumn{1}{c}{ Vantagens } & \multicolumn{1}{c}{ Desvantagens } \\
\hline $\begin{array}{ll}\text { - Taxas de inflação menores e mais estáveis } \\
\text { (países industrializados ad hoc); }\end{array}$ & $\begin{array}{l}\text { • não há evidências de que sejam obtidas } \\
\text { taxas de inflação menores e mais estáveis } \\
\text { para o caso de países emergentes; }\end{array}$ \\
$\begin{array}{ll}\text { - disciplina dos gastos governamentais devido } \\
\text { à queda na captação de senhoriagem; }\end{array}$ & $\begin{array}{l}\text { - política monetária não é utilizada de forma } \\
\text { contracíclica; }\end{array}$ \\
$\begin{array}{ll}\text { - concentração do objetivo da política monetária } \\
\text { na busca pela estabilidade de preços; }\end{array}$ & $\begin{array}{l}\text { • não impede o aumento do déficit fiscal via } \\
\text { redução da taxa de juros; }\end{array}$ \\
$\begin{array}{l}\text { - redução do viés inflacionário na condução da } \\
\text { política monetária; }\end{array}$ & $\begin{array}{l}\text { - não é uma estrutura democrática; } \\
\text { - redução de interferência de natureza política nas } \\
\text { decisões operacionais do BC. }\end{array}$ \\
\hline
\end{tabular}

\section{PRÓS E CONTRAS DA COORDENAÇÃO DE POLÍTICAS}

É importante observar que apesar da teoria que analisa o problema de credibilidade da política monetária apresentar uma consistência interna apreciável, deve-

\footnotetext{
${ }^{4}$ Não obstante a idéia de que a presença de um BCI estaria associada a uma estrutura não-democrática, existe a possibilidade de compatibilizar um BCI e democracia. Blinder (1998) ressalta seis pontos nessa direção: i) o fato de algumas decisões dependerem mais da restrição imposta pela Constituição do que de ações tomadas no dia-a-dia do Legislativo, impede que decisões básicas sejam revistas com frequiência; ii) as metas estabelecidas para o $\mathrm{BC}$ devem ser definidas por políticos eleitos e não por tecnocratas; iii) o público tem o direito de exigir honestidade dos responsáveis pelo $\mathrm{BC}$ em troca do poder que lhes é concedido; iv) o presidente do BC deveria ser indicado via ação política; v) autoridades políticas poderiam, em casos extremos, refutar as decisões tomadas pelo BC; e vi) a necessidade de transparência e responsabilidade nas ações tomadas pelo BC.
} 
-se chamar atenção para o fato de que a base que sustenta a teoria não é sólida. Para que o exemplo criado por Kydland \& Prescott (1977) e posteriormente desenvolvido por Barro \& Gordon (1983) faça sentido, é assumido que a existência do viés inflacionário deriva do fato de o $\mathrm{BC}$ pactuar com o governo na busca de uma taxa de desemprego abaixo da natural, e que para alcançar este objetivo faz uso do imposto inflacionário. Ou seja, há muitas hipóteses ad hoc para a validade da teoria, o que indica ser mais adequado entender grande parte da literatura sobre a credibilidade da política monetária como um caso particular, e por conseguinte, deve-se ponderar de forma criteriosa a sua aplicabilidade ao mundo real.

Além disso, a teoria que advém do problema do viés inflacionário pressupõe o mau gerenciamento da política monetária. $\mathrm{Na}$ tentativa de solucionar este problema foram desenvolvidas duas estruturas básicas que não foram capazes de apresentar uma solução ótima: o conservadorismo do central banker ${ }^{5}$ e a aplicação de contratos ótimos sob a teoria do agente-principal. No período recente, a literatura tem apontado o uso de metas inflacionárias como um importante mecanismo para assegurar a independência operacional do BC. Não obstante, é importante que não seja desprezada a necessidade de a política monetária ser conduzida com a preocupação de evitar flutuações não só da inflação, mas também do produto.

Deve-se lembrar que do ponto de vista teórico não há consenso entre os economistas de que a única variável que a longo termo pode ser afetada pela política monetária é a taxa de inflação.

"Para darmos apenas alguns exemplos, Robert Eisner, ex-presidente da American Economics Association e renomado especialista em macroeconomia nunca aceitou a concepção de Friedman-Lucas. Tampouco ela foi aceita por James Tobin, Paul Samuelson, Robert Solow ou pelo finado William Vickrey - todos eles ganhadores do prêmio Nobel. O mesmo se deu com Ray Fair, de Yale; James Medoff, de Havard; e William Dickens, da Brookings Institution. [Além disso], Alan Greenspan, Alice Rivlin e os outros dirigentes do Federal Reserve... [têm] demonstrado quão espúria é a doutrina da taxa natural ao provar que pleno emprego, crescimento equilibrado e uma estabilidade de preços razoável não são mutualmente exclusivos." (Galbraith, 1999, p. 24, 25)

É diante desse cenário que não deve ser desprezada a possibilidade de coordenação de políticas macroeconômicas como uma alternativa factível para estruturas teóricas que recomendam a concentração de políticas econômicas em um objetivo. A justificativa para este comportamento é simples. Em sociedades democráticas, o cidadão elege seus representantes na expectativa que sejam alcançados diversos objetivos pelo governo de forma que possa ser alcançado o melhor resultado possível em termos de bem-estar. Como a taxa de inflação representa apenas um dos

\footnotetext{
${ }^{5}$ Há dois problemas básicos relacionados à esta solução. i) a presença de um central banker conservador impõe preferências para a condução da política monetária diferentes da do público, o que representa uma estrutura não-democrática; e ii) no longo termo, um BC não pode operar sem o apoio do público. (Mishkin, 2000)
} 
elementos que o público leva em consideração quando da escolha de seus candidatos, não é justo que, após eleito, o representante da sociedade dê prioridade a um objetivo que possa prejudicar o alcance das demais.

$\mathrm{O}$ argumento acima não implica a negação de que a inflação seja um objetivo de política relevante, mas que ele não é o único. Ademais, nenhum economista rejeita o fato de que alterações nas taxas de juros da economia sejam capazes de afetar a atividade econômica. Assim, deve-se, no mínimo, ter o cuidado de observar se o uso da política monetária na busca do difundido objetivo da busca da estabilidade de preços não está acarretando custos sociais desnecessários (aumento do déficit, desemprego, etc.).

O significado do que é coordenação tem sido utilizado de forma peculiar por autores que adotam a premissa da neutralidade da moeda. Goodhart (1995) é um bom exemplo de como defensores da IBC deturparam o conceito de coordenação de políticas. De acordo com o autor supracitado, a base para o entendimento do que é coordenação (fictícia) ${ }^{6}$ corresponde à subordinação de uma política em relação à outra em função de qual delas é implementada primeiro. Nesse sentido, a coordenação (fictícia) seria resultado de uma espécie de jogo onde o BC teria o privilégio de mover-se primeiro e caberia à autoridade fiscal a responsabilidade de manter o orçamento equilibrado. Assim, ao invés de a presença de um BCI representar um entrave à coordenação (ficitícia), haveria um incentivo à consolidação da mesma. Nas palavras de Mishkin (2000, p. 5)

“(...) a government commitment to price stability also is a commitment to making monetary policy dominant over fiscal policy, ensuring a better alignment of fiscal policy with monetary policy."

É importante ressaltar que a idéia de coordenação (fictícia) fiscal-monetária, a partir de um BCI, não apresenta uma solução eficiente, pois por meio de uma coordenação (de facto) é possível alcançar um maior nível de bem-estar social. Ademais, o argumento de que BCIs são capazes de promover uma política fiscal mais equilibrada deve ser observado com cuidado. É verdade que na presença de uma autoridade monetária que não permita a captação de senhoriagem pelo governo há uma contenção dos gastos públicos. Todavia, o uso de uma política monetária restritiva implica taxa de juros mais elevadas. Este fenômeno é de crucial importância, pois uma taxa de juros mais elevada tem um duplo papel:

i) inibe investimentos - à medida que a taxa real de juros se eleva, os investimentos, cujos retornos tornaram-se inferiores ao que seria obtido pela aplicação dos recursos no mercado financeiro, tornam-se economicamente inviáveis podendo acarretar uma queda no nível de investimento da economia; e

ii) aumento do déficit - à medida que a taxa de juros aumenta, maior é a possibilidade de que ocorra um incremento do déficit, visto que independente do

\footnotetext{
${ }^{6}$ Como a interpretação de coordenação utilizada por Goodhart (1995) corresponde a uma situação específica de coordenação em que há a preocupação de torná-la favorável à adoção de um BCI, achouse conveniente utilizar o termo - coordenação fictícia - para este caso.
} 
controle dos gastos no período atual, a taxa de juros incide sobre o estoque da dívida do período anterior. Destarte, um resultado primário equilibrado é insuficiente para que seja evitado um aumento progressivo do déficit na economia.

Portanto, um importante argumento a favor da coordenação de políticas refere-se ao risco potencial que uma política monetária contracionista na busca da estabilidade de preços pode acarretar sobre o déficit e o desemprego e, ainda, a possibilidade de retorno da inflação no longo termo decorrente da possibilidade de uma trajetória insustentável para o endividamento público.

A análise realizada acima tem como variável-chave as possíveis implicações negativas oriundas da situação de dominância monetária. Além deste caso, um outro argumento pró-coordenação pode ser identificado a partir dos problemas provenientes do caso de dominância fiscal. Sob esta perspectiva, há a tendência de que o responsável pela política não mantenha o equilíbrio fiscal devido à expectativa de que o BC garantirá os recursos necessários para satisfazer os gastos realizados. Assim sendo, o provável resultado de uma dominância fiscal é o aumento do déficit em decorrência do aumento dos gastos e o aumento da taxa de inflação derivado da emissão monetária pelo BC. ${ }^{7}$

\section{Esquema 2}

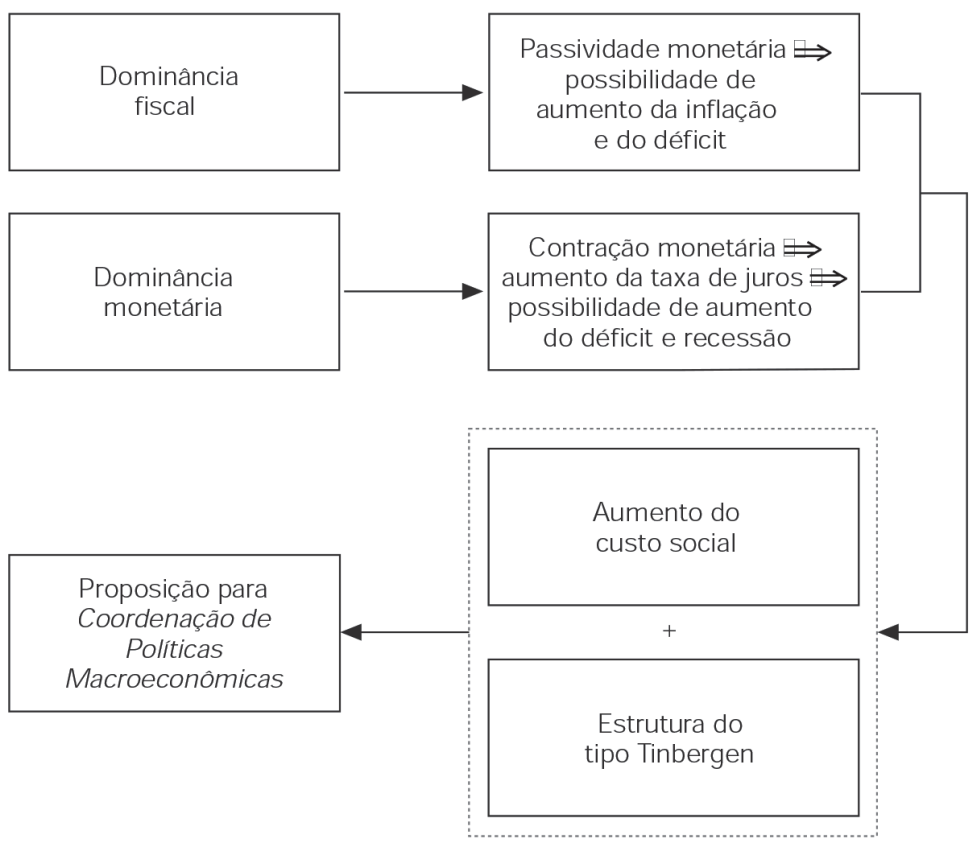

\footnotetext{
${ }^{7}$ Tal como ressaltado pelos defensores da IBC, com base na conhecido argumento desenvolvido por Sargent \& Wallace (1981), é reconhecido que a dominância fiscal também é um problema para que a coordenação de políticas torne-se factível.
} 
O esquema 2 sintetiza os principais argumentos que justificam o porquê da adoção de uma estrutura com base na coordenação de políticas macroeconômicas. Deve-se ressaltar que o desenvolvimento teórico para a análise de coordenação de políticas deve estar respaldado na estrutura inicialmente desenvolvida por Tinbergen (1952). Em outras palavras, é preciso que seja admitida a possibilidade da política monetária ter a capacidade de afetar outras variáveis que não seja apenas a taxa de inflação, pois dessa forma é possível encontrar soluções que impliquem maior nível de bem-estar social.

Deve-se ressaltar que a regra de reação apresentada por Taylor (1993) para a determinação da taxa de juros básica da economia, representa um aparato razoável para a proposição de coordenação de políticas. A estrutura proposta pelo autor supracitado é compatível com a desenvolvida por Tinbergen (1952). Ademais, é explícito o uso da política monetária para a busca da estabilidade de preços, mas há a preocupação de que seja evitado um movimento recessivo sobre a economia. As evidências empíricas encontradas por Mendonça (2001) para o caso brasileiro sugerem que a adoção desta estrutura seria capaz de manter o regime de metas para a inflação, reduzir a taxa de juros e contribuir para a redução do déficit.

Não obstante os bons resultados que podem ser extraídos a partir da coordenação de políticas, o principal entrave à sua adoção refere-se à dificuldade em avaliar o tempo que a ação da política monetária leva para afetar as expectativas e decisões dos agentes e, ainda, as conseqüências para o lado real da economia. Contudo, esses são problemas que são observados no uso do regime de metas inflacionárias e nem por isso tem havido desestímulo ao uso dessa estratégia por diversos países.

Como os problemas relacionados à coordenação de políticas macroeconômicas são semelhantes àqueles referentes à aplicação de metas para inflação e os resultados obtidos são potencialmente superiores (a política monetária é conduzida de forma que sejam evitadas grandes flutuações sobre a inflação e o produto), pode-se afirmar que esta alternativa de condução de política econômica é factível e há maior nível de bem-estar social. Além disso, conforme reconhecido por Barro (1986, p. 35-36, grifo meu)

"The choice between the two objectives - stabilising the general price level versus stabilising nominal GNP - corresponds to the weights one attaches to the validity of the two competing viewpoints about macroeconomics. (Surely one of these views must be correct!)"

Destarte, por que insistir apenas na primeira opção? A coordenação de políticas macroeconômicas tem a vantagem de considerar os dois objetivos. Na verdade, os dois propósitos são relevantes, não há motivos a priori para que a política monetária concentre-se em apenas um deles.

Pode-se dizer que a coordenação de políticas é superior ao caso de independência, pois permite um melhor planejamento dos objetivos macroeconômicos. Além disso, no caso de uma acentuada desacelaração (ou aceleração) do produto ou da inflação, a ação conjunta das políticas permite direcionar a economia para o equilíbrio planejado. Por outro lado, no caso onde não há coordenação emerge 
a possibilidade de conflito entre as autoridades monetária e fiscal, o que implica uma fonte potencial de custos sociais.

Deve-se ressaltar que não se deve associar à coordenação entre as políticas monetária e fiscal a idéia de desleixo para os diversos objetivos do governo (inflação, desemprego, etc.). Em outras palavras, coordenação não é sinônimo de passividade monetária ou fiscal. Na verdade, a coordenação associada à transparência na condução das políticas representa uma estrutura capaz de reduzir o nível de incerteza contribuindo para uma formação de expectativas positivas dos fundamentos econômicos.

\section{CONCLUSÕES}

Deve-se ressaltar que a proposição de IBC apresenta uma base teórica que não se mostra adequada para que se possa considerar um BCI como uma estrutura apropriada para qualquer economia, a principal evidência empírica que dá sustentação a esta proposição é no mínimo curiosa. A propalada conclusão de que BCIs implicariam menores taxas de inflação só é observado para o caso de economias industrializadas, o que, por conseguinte, sugere que não é uma estrutura adequada para que economias emergentes encontrem o caminho da estabilidade. Por outro lado, a proposta de coordenação de políticas além de permitir a conquista de credibilidade do BC, pode, por meio da aplicação da regra de Taylor, fazer uso do principal estratégia para a condução da política monetária presente nos modelos de terceira geração de $\mathrm{IBC}^{8}$ - a aplicação de metas para inflação. Ademais, a coordenação obriga o BC a executar uma política monetária mais responsável não só em termos de inflação, mas também de forma a impedir recessões e aumento do déficit de forma desnecessária.

Uma importante observação a ser feita é que o esforço empregado por diversos economistas para o encontro de um mecanismo capaz de assegurar a estabilidade de preços sem custos sociais não é adequado. A separação propalada entre o BC e o governo é ilusória. De certa forma, o reconhecimento de que o $\mathrm{BC}$ deve ter apenas independência operacional concomitante à aplicação de metas representa um claro sinal de que existe um limite para a separação entre o governo e o BC. ${ }^{9}$

A transformação crucial na relação governo-BC deve-se à criação de mecanismos (contratos) com o objetivo de evitar que o BC desvie-se daquilo que foi previamente acordado com o governo, contudo, a essência continua a mesma - o governo (representante da sociedade) determina quais devem ser os objetivos a serem alcançados em termos de inflação e o BC atua como órgão executor.

Um ponto fundamental é o fato de que não é desprezível a possibilidade da política monetária ter efeitos duradouros, e que, portanto, os impactos sobre o

\footnotetext{
${ }^{8}$ A respeito da taxonomia dos modelos de IBC, ver Mendonça (2000).

${ }^{9}$ É importante ressaltar que a observação acima não implica uma negação, por parte do autor, da utilidade de elementos como: maior transparência, uso de metas, etc.
} 
lado real da economia não devem ser entendidos apenas como efêmeros. Dado que o objetivo principal de um governo deve ser a maximização do bem-estar da sociedade, e que os objetivos de política econômica não se limitam apenas à inflação baixa, a coordenação de políticas econômicas representa um aparato poderoso para o alcance das diversas metas pretendidas pelo governo.

\section{REFERÊNCIAS BIBLIOGRÁFICAS}

ALESINA, A. \& SUMMERS, L. (1993) “Central Bank Independence and Macroeconomic Performance: Some Comparative Evidence" Journal of Money, Credit and Banking, May.

BARRO, R. J. (1986) "Recents Developments in the Theory of Rules Versus Discretion” The Economic Journal, 96.

BARRO, R. J. \& GORDON, D. (1983) "Rules, Discretion and Reputation in a Model of Monetary Policy" Journal of Monetary Economics, 12, North-Holland.

BLINDER, A.S. (1998) Central Banking in Theory and Practice. Cambridge, MA: MIT Press.

CUKIERMAN, A. (1994) “Central Bank Independence and Monetary Control” The Economic Journal, 104, November.

GALBRAITH, J.K. Fixados na Inflação. Foreign Affairs. Gazeta Mercantil, 1999.

GOODHART, C. (1995) Central Banking and Financial System. London: MacMillam.

HAHN, J. \& STURM, J. (1992) “The Case of Central Bank Independence” Banca Nazionale del Lavoro Quarterly Review, September.

KYDLAND, F. E. \& PRESCOTT, E. C. (1977) "Rules Rather than Discretion: the Inconsistency of Optimal Plans" Journal of Political Economic, 85 (3).

LUCAS Jr., R. (1972) “Expectations and the Neutrality of Money" Journal of Economic Theory, 4. . (1973) "Some International Evidence on Output-Inflation Tradeoffs" The American Economic Review, June.

MENDONÇA, H.F. (2001) “Coordenação de Políticas Macroeconômicas: Implicações para o Caso Brasileiro”. In: Finanças Públicas: V Prêmio Tesouro Nacional. Brasília: ESAF. . (2000) "A Teoria da Independência do Banco Central: Uma Interpretação Crítica” Estudos Econômicos, Instituto de Pesquisas Econômicas - USP, São Paulo, 30 (1).

. (1998) "Aspectos Teóricos e Empíricos sobre Bancos Centrais Independentes: Implicações para o Caso Brasileiro" Economia Aplicada, FIPE/FEA-USP, São Paulo, Jan/Mar, 2 (1) (Prêmio Brasil de Economia).

MISHKIN, F. (2000) “What Should Central Banks Do?” Review. Federal Reserve Bank of St. Louis, V. 82 (6), November/December.

MUNDELL, R. (1960) “The Monetary Dynamics of International Adjustment under Fixed and Flexible Exchange Rates" Quarterly Journal of Economics, May.

NORDHAUS, W.D. (1994) "Policy Games: Coordination and Independence in Monetary and Fiscal Policies”. Brooking Papers on Economic Activity, V. 2. William C. Brainard and George L. Perry (ed.). The Brookings Institution, Washington.

ROGOFF, K. (1985) “The Optimal Degree of Commitment to an Intermediate Monetary Target” The Quarterly Journal of Economics, November.

SARGENT, T.J. (1973) "Rational Expectations, the Real Rate of Interest, and the Natural Rate of Unemployment" Brooking Papers on Economic Activity.

TAYLOR, J.B. (1993) “Discretion Versus Policy Rules in Practice”. Carnegie-Rochester Conference Series on Public Policy, N. 39.

TINBERGEN, J. (1952) On the Theory of Economic Policy. North-Holland. 